NOTAS Y DISCUSIONES

\title{
Contribuciones del utilitarismo contemporáneo a la ética de las decisiones públicas
}

\author{
JULIA BARRAGÁN \\ Universidad de Caracas
}

\section{Introducción}

En el riquísimo campo de la discusión ética el tratamiento de la corriente de pensamiento conocida en general como utilitarismo se ha desarrollado frecuentemente en escenarios tormentosos, que muchas veces no son sino el resultado de prejuicios alimentados tanto por quienes adversan al utilitarismo como por aquellos que asumen su defensa. Éste es un caso más en el que se evidencia la malévola influencia que los prejuicios son capaces de ejercer sobre una discusión, influencia especialmente nociva y dificil de evitar porque los tales prejuicios no suelen presentarse a cara descubierta; la mayoría de las veces usan espesos velos que logran disimular su verdadero perfil, y en no pocas oportunidades su rostro se hace completamente irreconocible envuelto en ricos y seductores ropajes argumentales. Hay que dejar en claro que por lo general ni los espesos velos ni los vistosos ropajes son utilizados con el fin deliberado de ocultar o disimular el verdadero perfil de los prejuicios; sin embargo, más allá de la voluntad de quienes con ellos se relacionan, los mismos suelen ser portadores de una fuerte capacidad para imponerse sobre cualquier intención esclarecedora y de una singular fuerza que convicrte en extremadamente dificil el avance por el siempre estrecho y sinuoso desfiladero de la elaboración de soluciones constructivas. Para el logro de una evaluación equilibrada de las eventuales contribuciones del utilitarismo contemporâneo al campo de las decisiones públicas, hay un prejuicio que produce importantes interferencias; se trata de la creencia, frecuentemente alimentada por muchos políticos y no pocos filósofos, de que cuando un decisor público «toma una decisionn» lo hace sobre la base de un conocimiento claro que más allá de toda duda le señala sin ambigüedades ni condicionalidades cuál es el bien general; esto parece dotar al decisor de plena certidumbre acerca de la corrección de su decisión. Desde este prejuicio es difícil apreciar y menos aún atribuir importancia a los siempre pequeños logros dirigidos a disminuir la incertidumbre o a corregir mínimos sesgos de decisión.

Inicialmente, y sin entrar aún a juzgar su importancia, es un hecho cierto que el camino recorrido por el pensamiento utilitarista durante el siglo $\mathrm{xx}$ evidencia una búsqueda sostenida de precisión en los conceptos. Esta búsqueda permitirá ir sustituyendo expresiones tan vagas como las de placer, dolor o felicidad por definiciones con una mayor capacidad de delimitación, y que en consecuencia están en condiciones de ofrecer un piso más sólido para 
la toma de decisiones públicas. Por otra parte, las formas incipientes de cálculo que se perfilaron en las obras de Bentham ${ }^{1}$ cedieron el lugar a estructuras de mayor complejidad y con un fuerte poder para efectuar derivaciones. Estas propiedades han hecho posible que la tarea de decidir en el ámbito público pueda ser practicada con mayor sutileza y al mismo tiempo que los decisores estén en condiciones de ofrecer razones más transparentes para justificar sus decisiones. Este hecho desde luego posee connotaciones instrumentales bien evidentes; pero sus efectos se proyectan más allá del campo estrictamente práctico, llegando a incidir notablemente en la capacidad normativa de las asignaciones deónticas y distributivas que subyacen a toda decisión pública.

Analizar desde esta óptica las contribuciones del utilitarismo contemporáneo a la ética de las decisiones públicas puede ayudar notablemente al enriquecimiento y complejización de la discusión intelectual en la materia, y al mismo tiempo servir a importantes aspectos prácticos de la vida social que se vinculan con la Ética.

\section{Los componentes de las decisiones públicas}

En toda decisión pública es posible diferenciar al menos dos componentes sustantivos, los cuales, aunque se interrelacionan de manera estrecha, poseen esquemas de validación perfectamente diferenciables, responden a legalidades diversas y son separables analíticamente. Uno de los aspectos señalados es el relacionado con los instrumentos que la decisión utiliza para asignar los beneficios y cargas sociales a los que se refiere; el segundo aspecto se vincula a la eficacia normativa de la decisión, es decir, a su capacidad para convocar la adhesión de sus destinatarios.

El primero de los aspectos requiere contar con un sistema de axiomas que señale los puntos de partida aceptados y de un cuerpo de reglas de derivación que serán las consideradas operacionalmente legitimas. Además de los obvios requerimientos lógicos que estos axiomas y reglas deben satisfacer, ellos deben ser evaluados en términos de su plausibilidad y potencia explicativa en relación con el problema específico de decisión al cual serán aplicadas. Esto último se debe a que las decisiones son directamente alteradas por el instrumental que se utiliza en su construcción; o lo que es lo mismo, las decisiones dependen en buena medida del sistema formal con el cual son elaboradas ${ }^{2}$. De esto nace la necesidad de justificar suficientemente el uso de un determinado sistema de supuestos metodológicos, lo cual implica la exclusión del uso de otro sistema alternativo.

Pero aun cuando se realice la más cuidadosa selección de los componentes instrumentales de una decisión pública, éstos sólo alcanzan para garantizar la construcción de una decisión técnicamente sostenible y correcta dentro de los marcos previamente establecidos, lo cual es una condición necesaria pero no suficiente para justificar plenamente dicha decisión. El segundo componente de las dccisiones públicas al que hemos denominado «eficacia normativa» se refiere a la capacidad real de tales decisiones para generar espacios de convivencia y para hacer menos costosas las transacciones intersubjetivas. Aunque la eficacia normativa requiere de una sólida construcción instrumental, ésta cs incapaz por sí sola de generarla, por cuanto aquélla está íntimamente asociada con factores de legitimidad que rebasan los aspectos instrumentales. Tales factores derivan de la transparencia con que las decisiones públicas son elaboradas, condición ésta que se vincula a la confiabilidad de los esquemas argumentales usados en su fundamentación y a la sustentabilidad de las asignaciones deónticas que la decisión produzca. 
En este punto hay que considerar que las decisiones públicas que establecen las asignaciones deónticas son tomadas bajo condiciones de incertidumbre, la cual deriva de diversas fuentes. Para mencionar sólo algunas se podrían señalar las derivadas de los fines, los cuales pueden postular la deseabilidad de diferentes valores; por ejemplo, en algunos casos, fines altamente valiosos en el largo plazo no lo son en el corto plazo; y es extremadamente difícil para el decisor determinar cuál es el limite entre uno y otro plazo, y en consecuencia cuán valioso es cada fín alternativo. Otro factor que también genera incertidumbre deriva del carácter autónomo de los sujetos morales, los cuales gozan de plena libertad para moverse en un amplio abanico de comportamientos, y pueden plantearle al decisor verdaderos dilemas tanto materiales como morales. En este escenario de incertidumbre, la eficacia normativa no recibe del terreno de la legalidad juridica un soporte inexpugnable, aunque su apoyo sea necesario. Esto se debe a que en esta arena no se trata de discutir acerca de si la decisión fue elaborada consistentemente dentro de tal o cual marco legal, sino de juzgar cuál es su capacidad para resolver exitosamente las profundas discrepancias entre las funciones de utilidad individual y las de utilidad colectiva que se despliegan, a veces dramáticamente, en el escenario de la decisión pública. Tampoco las asignaciones deónticas incondicionales ofrecen garantías suficientes para sostener la eficacia normativa, ya que los marcos de las decisiones bajo incertidumbre son condicionales por definición, y el nivel de realizabilidad de una solución depende básicamente de las condiciones del entorno ${ }^{3}$. Los mecanismos para potenciar la capacidad normativa de una decisión pública son menos contundentes y monolíticos. Ellos se asocian a condiciones măs vagas tales como la transparencia con que el decisor despliega ante los usuarios de la institución sus propias estructuras decisionales, despliegue éste que permite a los últimos captar la intencionalidad de la decisión y eventualmente compartirla o no. Asimismo, los citados mecanismos se vinculan a la consistencia, tanto lógica como valorativa, de la base argumental de la decisión, la cual provee a los usuarios de la oportunidad de evaluar las consecuencias del arreglo normativo propuesto, $y$ de este modo acordar o discrepar racionaimente sobre el mismo. Finalmente, como en toda decisión pública se produce una asignación de cargas y de beneficios en relación con bienes comunes, la misma debe ser sustentable como solución, tanto en términos de la calidađ de la asignación como de su potencial estabilidad.

En el marco específico de las decisiones públicas, que como se ha visto es un marco incierto y sembrado de dificultades, no parece haber reglas algorítmicas para la decisión, sino estrechos cursos de acción a través de los cuales es posible llegar a una solución más defendible o más estable en un entorno determinado. Considerando analíticamente algunos de los principales hallazgos del utilitarismo contemporáneo se puede observar que en este marco de incertidumbre ellos han logrado mejorar la calidad de los aspectos instrumentales $y$ han contribuido a reforzar de manera considerable la eficacia normativa, al generar un espacio más transparente para la construcción de las decisiones públicas.

\section{Una contribución de importancia al mejoramiento del componente instrumental}

La regla de decisión del utilitarismo goza de una gran popularidad (tal vez sólo comparable con la de la regla de oro kantiana); aun quienes ignoran casi todo acerca del llamado utilitarismo, tanto en su vertiente clásica cono contemporánea, al ser preguntados por la regla del utilitarísmo no 
dudan en responder que ella indica «maximizar.....". Aquí los puntos suspensivos pueden ser sustituidos por diversos conceptos. Algunos hablarán de la felicidad, otros del placer, habrá quienes se inclinen por el bienestar y unos más por las ganancias, con lo que la regla de maximización pasa a tener múltiples contenidos (es decir, sc convjerte en muchas reglas y en ninguna), multiplicidad que alimenta un gran número de polémicas acerca de cuál es el contenido correcto. Le corresponde al utilitarismo contemporáneo el importante mérito de haber afinado la definición de una única regla de decisión, la cual indica que el agente optarấ por la «maximización de las utilidades esperadas». La construcción de esta regla ha supuesto un arduo trabajo dirigido en primer lugar a la búsqueda de una definición de la estructura primitiva de la Teoría de la Utilidad, que incluye su tratamiento axiomático; dicho trabajo se ve satisfactoriamente coronado cuando se alcanza una regla única. A pesar de este carácter único, la polivalencia de la regla permite que la misma pueda ser aplicada a diversos niveles de decisión, incluidos aquellos que versan sobre las reglas morales.

En lo relativo a hallazgos que conducen al perfeccionamiento de los aspectos instrumentales, el utilitarismo contemporáneo ha logrado un gran avance al precisar su estructura primitiva ${ }^{4}$ estableciendo por una parte los llamados presupuestos y por la otra efectuando el tratamiento axiomático del concepto de utilidad. Este logro ha contribuido grandemente a dotar a la regla de decisión elaborada por el utilitarismo contemporáneo de rasgos ciertamente deseables. Desde un punto de vista teórico se ha logrado una regla intersubjetivamente criticable y con mecanismos claros de corrección, y desde un punto de vista práctico lal regla ha demostrado ser capaz de orientar las dccisiones con menor ambigüedad y un más alto poder de discriminación entre los diferentes casos.
Veamos en primer lugar cómo ha opcrado este proceso. Desde el punto vista de la Filosofía de la Ciencia se dice que una teoría está lógicamente reconstruida cuando ha logrado la formulación simbólica de sus axiomas y el pleno establecimiento de sus supuestos y reglas. Es decir, cuando su estructura primitiva está plenamente definida. Curiósamente, el mérito de haber contribuido inicialmente $y$ de manera decisiva a la definición de la cstructura primitiva del utilitarismo debe ser atribuida a dos pensadores que hasta donde es conocido nunca mostraron especial interés intelectual por el utilitarismo; ellos son John von Neumann y Oskar Morgenstern, quienes en su obra Theory of Ganes and Economic Behavior, publicada en 1944 por Princeton University Press ${ }^{5}$, emprenden y culminan exitosamente una tarea cuyo singular valor lógico y matemático sólo puede ser comparado con el profundo significado que más tarde adquiriría en el terreno fillosófico ${ }^{\circ}$.

La firme convicción de Von Neumann y Morgenstern acerca de la plausibilidad de una medición cardinal de las preferencias les mantiene por cncima de la polémica que desde los años treinta había dividido al mundo de los economistas en ordinalistas versus cardinalistas. En rigor, mientras tal polémica se escenificaba, ellos establecían en su obra los requisitos formales bajo los cuales la medición cardinal de las preferencias es posible. Nace así el concepto de la utilidad como medida cardinal de las preferencias.

El desarrollo del concepto de Von Neumann y Morgenstern por un camino análogo al seguido en las curvas de indiferencia avanza en el sentido de establecer no sólo un ordenamiento de las preferencias, sino también los valores relativos de las mismas. Como es natural, el uso de la scńalada herramienta hace que los requisitos formales que demanda la mediciôn numérica de las preferencias estén 
asociados a los establecidos para el análisis de las curvas de indiferencia.

El primer requisito establecido por Von Neumann y Morgenstern es que el decisor posea un sistema de preferencias, $o$, lo que es lo mismo, que ante una situación determinada esté en capacidad de ordenar tales preferencias. Esto quedaría expresado en el hecho de que en una situación determinada y ante dos objetos o resultados ( $\mathrm{A}$ y B) él estê en condiciones de preferir uno al otro, o bien que pueda establecer que es indiferente entre ambos. Por otra parte, este requisito también involucra que si $\mathrm{A}$ es preferido a $\mathrm{B}$ y $\mathrm{B}$ a $\mathrm{C}$, entonces $\mathrm{A}$ es preferido a $\mathrm{C}$.

El segundo requisito demanda que el decisor esté en condiciones de escoger coherentemente entre loterias de objetos o resultados; por ejemplo, preferir A sobre una lotería de $\mathbf{B}$ y $\mathbf{C}$, o inversamente, o bien ser indiferente entre $\Lambda$ y la lotería By C?

Los requisitos establecidos por Von Neumann y Morgenstern, como toda estipulación, suponen definir restricciones que fijan los límites dentro de los cuales la teoría en cuestión puede ser interpretada; toda interpretación intentada fuera de tales límites no sólo debe ser calificada de ilegítima, lo cual a nuestro juicio constituye un asunto de relevancia menor, sino que, y esto sí es crucial, tal interpretación carecería de capacidad para formular una crítica consistente que pudiera contribuir a fortalecer o a refutar el enfoque; es decir, habría perdido toda significación teórica. Esto significa que si nos encontramos frente a un actor que no satisfaga los requisitos señalados, es decir, un actor que ante una situación dada sea incapaz de ordenar coherentemente sus preferencias, tendríamos para tal caso específico que renunciar a esta teoría de la utilidad.

Aun cuando éste no sea el momento exacto para desplegar con amplitud argumentos sobre el punto, quisiera expresar un comentario acerca de la plausibilidad de estos requisitos; me gustaría llamar la atención sobre algunas críticas que a propósito de este punto se suelen efectuar. Se habla con frecuencia de que estas restricciones de Von Neumann y Morgenstern son excesivas, y que prácticamente ningún humano está en condiciones de satisfacerlas, por lo que la teoría quedaría sin referentes de aplicación; quienes así se expresan no parecen advertir el efecto que tal afirmación produce sobre el concepto de autonomía (tan importante para la Ética). En efecto, si un agente no es capaz de ordenar consistentemente sus preferencias, ya sea que ellas estén dictadas por la pasión o por la razón, parece que estamos en presencia de un incompetente básico cuya autonomía para decidir es francamente cuestionable; y si tales agentes son, como pretenden tales críticos, una gran mayoria, el conjunto de los sujetos morales quedaría dramáticamente disminuido; este hecho tendría que generar una profunda preocupación no sólo entre los seguidores del utilitarismo, sino también entre sus críticos; y desde luego entre todos quienes se preocupan por los problemas éticos, y hasta me atrevería a decir que en el seno de la humanidad toda.

El tratamiento axiomático de la utilidad se ha ido desarrollando paso a paso; en este aspecto se ha utilizado el ya clásico método de establecer un sistema de axiomas, y luego de evaluar su capacidad para generar consecuencias, enfrentarlo a un sistema rival (por lo general, sólo se trata de sustituir algún axioma específico) a fin de establecer mediante comparación su nivel de fortaleza .

El sistema axiomático establecido por Luce y Raiffa en Games and Decisions es por una serie de razones el favorito de un buen número de autores. Fstá constituido por seis axiomas, que junto a los supuestos de Von Neumann y Morgenstern ya señalados logran definir la estructura primitiva de la teoría de la utilidad. Sólo se hará una breve enunciación no formalizada de 
su contenido, ya que el propósito de este trabajo así lo aconseja:

Axioma 1. De ordenamiento de las alternativas. Para todo resultado A y $\mathbf{B}$ el decisor preferirá A sobre $B$; 0 bien $B$ sobre $A ; 0$ bien será indiferente entre ambos.

Axioma 2. De reducción de loterías complejas. Toda lotería compleja puede ser reducida a una simple mediante el cálculo de probabilidades.

Axioma 3. De continuidad. Sean los resultado $A, B$ y $C$. $S i$ es preferido a B y B a C, siempre existirá un valor $p$ (entre 0 y 1) tal que el decisor será indiferente entre B y una lotería con probabilidad $p$ de obtener el resultado $A$ y la probabilidad $(p-1)$ de obtener $\mathrm{C}$.

Axioma 4. De sustitución. Si dos loterías son indiferentes entre sí, entonces son intercambiables.

Axioma 5. De transitividad. La preferencia y la indiferencia entre loterías son relaciones transitivas.

Axioma 6. De monotonía. Entre dos loterías que involucran sólo la alternativa más y menos preferida, el decisor escoge el resultado que le ofrezca la mayor probabilidad esperada.

En el Axioma 6 se reconoce ya claramente la regla de decisión empleada hoy universalmente asociada al utilitarismo. Sin embargo, es importante señalar que esta regla no puede ser interpretada sino en el marco de los cinco axiomas precedentes. Así considerada resulta claro que el decisor, como un agente autónomo, puede reducir todos los componentes de un problema de decisión a loterías entre resultados, y podrá así evaluar la conveniencia de cada resultado mediante la probabilidad; pudiendo asimismo establecer comparaciones mediante un número real entre 0 y 1 . El decisor (y esto es consistente con los supuestos iniciales) se inclinará por la alternativa que le ofrezca una mayor utilidad esperada.
Como se puede observar, al asociarse las preferencias por determinados resultados con la probabilidad de que Ios mismos sucedan, ha nacido el finísimo concepto de «utilidad" como medida cardinal de las preferencias. Es evidente que los avances en el camino de alcanzar la definición de la estructura primitiva de la teoría de la utilidad han sido graduales, acumulándose cada vez pequeños hallazgos que permiten hoy exhibir un panorama más rico y complejo que en los inicios. Sin embargo, es legítimo preguntarse cuáles son, más allá de la elegancia formal, las ventajas de haber establecido tan cuidadosamente los mencionados presupuestos y el sistema de axiomas.

En primer lugar como un punto preliminar para evaluar la importancia de estos logros habria que señalar que, contrariamente a lo que muchos creen, las estructuras primitivas no representan el punto final y definitivo para el establecimiento de una teoría; tales estructuras son necesariamente corregibles (y mejorables), por lo que en rigor sería más acertado pensarlas como sólidos puntos de partida. El malentendido de considerarlas como un estadio final se nutre por 10 general en el hecho de que las estructuras formalizadas, por su elegancia y su capacidad de derivación, tienden a mostrarse como cuerpos inexpugnables al pensamiento crítico. Nada más alejado de la realidad que esta creencia; la historia es rica en ejemplos que muestran cómo monumentales aparatos axiomatizados como la Geometría de Euclides o el sistema de Hilbert fueron puestos en jaque por la labor de pensadores que consiguieron ampliar o diversificar las respucstas formales que aquéllos ofrecian.

El hecho de definir la estructura primitiva es en realidad una invitación al examen crítico riguroso, ya que de ese modo los supuestos y axiomas en lugar de encontrarse ocultos o desdibujados son colocados en una posición tal que quedan total- 
mente expuestos a cualquier forma de examen, tanto desde dentro como desde fuera del sistema. Adicionalmente, el tener una estructura primitiva bien definida contribuye a suministrar criterios estables de demostración y evaluación del comportamiento del sistema, lo cual permite contar con parámetros de corrección del mismo.

En el caso de la teoría de la utilidad es posible afirmar que desde el punto de vista metodológico el hecho de contar con un buen sistema de definiciones ha permitido dibujar un escenario en el que los esfuerzos críticos han producido a lo largo del tiempo resultados claramente constructivos. En cuanto al contenido sustancial plasmado en la regla de decisión que establece la maximización de la utilidad esperada, en primer lugar habría que señalar que la misma permite que la autonomía del agente se exprese a través del ordenamiento y la comparación intrapersonal de las preferencias; y en segundo lugar tal regla dota a ese agente autónomo de un criterio consistente con los supuestos establecidos acerca de su comportamiento. Por otra parte, el hecho de que las preferencias puedan ser medidas en «útiles» y no en unidades monetarias inflexibles o en conceptos vagos tales como los de felicidad o dicha, hace que las mismas sean capaces de expresar una gama extremadamente rica de información, tanto de origen subjetivo como objetivo. Esto se debe a que aunque el decisor trabaje con un conjunto limitado de alternativas en sus preferencias, al combinar tales alternativas con la probabilidad de ocurrencia de las mismas, el conjunto se hace teóricamente infinito, con lo que es posible realizar asignaciones $\mathrm{e}$ intercambios mucho más sutiles.

\section{La estructura de las decisiones normativas}

Toda decisión pública tiene origen en un acto de voluntad que atribuye un deter- minado status deóntico a un cierto tipo de acciones $\mathrm{u}$ omisiones o que realiza asignaciones distributivas de bienes comunes. Sobre la base del status establecido o de la asignación realizada, y con el fin de promover ciertos comportamientos y desestimular otros, las decisiones públicas definen asimismo un sistema de incentivos que tienden a premiar a quienes acatan las regulaciones establecidas y a castigar a aquellos que las transgreden; esto implica decir que toda decisión pública es normativa. Para cumplir eficazmente con su cometido, un arreglo normativo debe formular las asignaciones deónticas o distributivas de una manera clara, de modo que se disminuya la incertidumbre derivada de la formulación de la regla. Asimismo, mediante los mecanismos de incentivos debe procurar hacer onerosas las transgresiones de modo que la conducta de los sujetos sociales tienda a ser previsible, disminuyendo así la incertidumbre que se derivaría de una interacción no regulada.

Así considerada, en toda decisión pública se encuentran presentes tres elementos: por una parte está el agente de voluntad del cual emana la decisión al que llamaremos autoridad normativa ( $A N$ ); en segundo lugar se encuentra el arreglo normativo (N), que expresa dicha voluntad bajo la forma de mandatos, prohíbiciones, permisioncs o asignaciones distributivas dotadas de sus respectivos mecanismos de incentivos; finalmente se halla el sujeto o los sujetos (S) hacia los cuales va dirigida la decisión normativa, y cuyos comportamientos se verán afectados por la misma.

Se puede aceptar de manera general que uno de los objetivos más importantes de las decisiones públicas es el de hacer que las transacciones sociales sean lo más improblemáticas que resulte posible, lo que equivale a decir que en el sistema de relaciones interpersonales reguladas por una determinado arreglo normativo se produzca un bajo número de fricciones. Este objetivo ha sido denominado de disminu- 
ción de los costos de transacción; y en un mundo hipotético en el que todas las decisiones públicas cumplan plenamente con su objetivo, el costo de las transacciones sería igual a cero ${ }^{9}$.

Con el propósito de disminuir la incertidumbre y en consecuencia los costos de transacción, tanto los derivados de las reglas como de las interacciones que bajo tales reglas se produzcan, quienes tienen a su cargo la elaboración de las decisiones públicas cuentan con directrices acerca de algunos puntos que resultan claves para el logro de la señalada disminución. Tales directrices señalan, en términos generales, los frentes sobre los que el diseño de decisiones nomativas trabaja a fin alcanzar el objetivo de abaratar los costos de transacción.

En primer lugar, se acepta que el proceso de formación de la decisión normativa debe conducir a una clara fijación de los límites deónticos de las acciones que califica. Esto implica claridad en la formulación lingüistica de la regla, y consistencia lógica y semántica entre las diferentes reglas que definen el arreglo normativo. Es indiscutible que estos rasgos coadyuvan de manera definitiva a mermar ulteriores fricciones entre los destinatarios de la decisión ya que disminuyen la incertidumbre derivada de los significados de la regla y de la asignación distributiva. Las contribuciones en el campo instrumental que fueron señaladas en el apartado anterior han producido un efecto muy benéfico en materia de mejoramiento de la calidad formal de las reglas y las asignaciones.

Sin embargo, a pesar de que un arreglo normativo cuente con reglas claramente formuladas y consistentes, las fricciones derivadas de las relaciones interpersonales no llegan a ser completamente erradicadas. Es inevitable que en el juego de intereses que se escenifica en el marco de un arreglo normativo surjan controversias entre las partes, o bien que surjan contradicciones entre una parte y la decisión misma, bajo la forma de transgresiones. Más allá de toda duda, la dismimución de los conflictos y de las transgresiones es una meta clara en el panorama de las decisiones públicas; a pesar de la claridad de la meta, no hay acuerdo unánime acerca de cuáles son los mecanismos más eficientes para lograrla.

En el punto específico de la disminución de los conflictos hay un punto de vista que sostiene que un arreglo normativo debe prever reglas específicas que desalienten las controversias, $y$ que funcionen de manera automática en caso de discrepancias inevitables; desde este punto de vista el arreglo normativo debe estar listo para intervenir de manera directa cuando los intereses de los sujetos entren en disidencia. Ésta es una solución que aun cuando llegue a cumplir satisfactoriamente con sa cometido tiene un alto costo, no sólo presente, sino también futuro $\mathrm{y}$ potencial. Frente a esta solución, otra de menor costo operativo propugna que la decisión sólo diseñe escenarios y mecanismos de resolución de los conflictos, con lo cual la definitiva solución de los mismos dependerá no sólo del propio arreglo normativo, sino también de la capacidad negociadora y del poder que posean las partes en conflicto de intereses.

En lo relativo a las transgresiones, el purto es aún más complicado. Desde luego que un arreglo normativo que sea víctima de permanentes transgresiones no resulta concebible. Por ello, disminuir las transgresiones es un objetivo muy claro a ser perseguido; pero de nuevo tal claridad desaparece cuando se comienza a hablar de cuáles son los mecanismos más eficientes para alcanzarlo.

Una solución bastante directa al problema de cómo lograr que disminuya el número de transgresiones consiste en hacer que las conductas transgresoras sufran una sanción que las desestimule al punto que no se reiteren en absoluto o queden reducidas a su mínima expresión. 
Para alcanzar eficazmente tal objetivo la sanción debería cumplir con dos condiciones: en primer lugar, tendría que ser lo suficientemente fuerte como para constituir una pérdida que supere en monto las utilidades que eventualmente se logran a través de las transgresiones; y en segundo término, tendría que existir una alta probabilidad de que dicha sanción sea efectivamente aplicada a los transgresores.

\section{Es decir, que siendo:}

e: Los casos en los que un individuo comete una transgresión

$U$ (e): La utilidad que obticne tal individuo en los casos $e$.

S: La sanción que establecida para los casose.

$D(S)$ : La desutilidad que produce la sanción establecida para los casos $e$.

$p(\mathrm{~S})$ : La probabilidad de que S sea efectivamente aplicada en los casos $e$,

la relación eficiente para desestimular las transgresiones seria la siguiente:

$$
\mathrm{U}(e)<p(\mathrm{~S}) \cdot \mathrm{D}(\mathrm{S})
$$

Cuando un arreglo normativo establece sanciones en las que se prevé una fuerte punición para los que cometen transgresiones, pero la probabilidad de que tales sanciones sean efectivamente aplicadas es muy baja, la relación señalada no podrá nunca scr satisfecha, lo cual pone en evidencia que la importancia de los aspectos procedimentales y organizacionales tendentes a la eficiente aplicación de las normas son tan importantes para la relación (I) como el contenido punitivo de las mismas.

A pesar de la aparente simplicidad de la relación señalada en (I), el llevarla a la práctica supone numerosas dificultades no tan insignificantes. Las primeras dificultades surgen cuando hay que designar a los encargados de administrar el control y las sanciones; siempre hay actores que quedan disconformes con los árbitros pro- puestos, ya que, aun siendo muy buenos, su superioridad frente a otros con dotes semejantes sólo puede apoyarse sobre criterios más o menos vagos. La propia tarea de arbitrar es difícil de cumplir a pesar de su aparente sencillez; por otra parte, la condición de excelentes árbitros no implica que los mismos carezcan de criterios preformados, o de rasgos de flexibilidad, tolerancia, nobles sentimientos y aun sentimientos de repugnancia moral hacia algún tipo especial de transgresiones. Los sesgos que estos factores incorporan hacen que sea conveniente y aun necesario ejercer un control sobre los arbitros, y también responsabilizarlos por sus decisiones. Hay entonces que diseñar nuevos arreglos normativos y asimismo establecer nuevas sanciones, esta vez para punir a los árbitros que no cumplan eficientemente con su función. Todo esto contribuye, más allá de cualquier duda, a disminuir el número de transgresiones, pero, en virtud de los costos que el procedimento mismo genera, sería impropio afirmar que el mecanismo ha disminuido efectivamente los costos de transacción.

Esta solución directa, y aparentemente tan simple, ha probado ser también productora de altos costos de transacción. La necesidad de numerosos controles que se encadenan de manera sucesiva hacen notar que aumentar la probabilidad de aplicación de la sanción no es un hecho gratuito, ni siquiera barato, ya que demanda de estrategias que impidan la burla a tal aplicación, haciendo necesario mantener un complejo sistema de incentivos que se inmunice constantemente contra eventuales perversiones. Por otra parte el factor $\mathrm{S}$ (sanción) de la relación también ofrece problemas, ya que la posibilidad de aumento cle su intensidad no es ilimitada; es evidente que una sanción groseramente magnificada en relación con la magnitud de la falta cometida, aunque lograría mantener el sentido de la desigualdad (I), podría traer consecuencias francamente indesea- 
bles, además de ser conceptualmente insostenible.

De todo lo dicho se puede inferir que para que la relación (I) se mantenga como una desigualdad efectiva en un sentido colectivamente deseable, es fundamental lograr que la fuerza y la probabilidad de aplicación de la sanción sean suficientemente altas, lo cual supone una tarea ardua y permanente de detección y fortalecimiento de los múltiples aspectos que tienden a ser vulnerables o a pervertirse. Este método de control se fundamenta en la premisa de que el acatamiento colectivo se basa fundamentalmente en un punto de vista externo, to cual hace que sea altamente inestable.

A los señalados problemas de costos e inestabilidad habria que agregar que aun cuando se logre mantener una relación tal que haga poco rentable intentar la transgresión contra la decisión pública, dicha relación sólo puede garantizar una mayor cantidad y calidad de los recursos públicos (lo cual no es poco), pero no está en condiciones de garantizar, ni siquiera de promover por sí sola una solución cooperativa entre los miembros del grupo. Esto se debe a que el debilitamiento de la urdimbre normativa del colectivo, al que puede considerarse como el costo social de las reiteradas transgresiones y desilusiones, no constituye sólo un problema de eficiencia, sino de valores.

El alto costo y la falta de equilibrio de las soluciones controladas por la autoridad producen naturalmente desánimo; suelen dar la impresión de que se aborda una tarea que no sólo es inagotable, sino que a cada momento pone en evidencia su ineficacia. Ante esta situación inocultable, no es demasiado sorprendente que las soluciones que buscan apoyarse en las posibilidades que el individuo posee para revertir los señalados procesos perversos sean capaces de convocar un alto nivel de esperanzas. Para los que colocan todo el peso del control de las transgresiones sobre las capacidades individuales, el origen de las dificultades para mantener bajo control el volumen de las transgresiones se ubica en ciertos rasgos individuales de los miembros de cada colectivo. Quienes suscriben esta posición sostienen que el factor desencadenante de situaciones de descontrol normativo con altos costos de transacción se afinca en comportamientos personales proclives a ceder al vicio del egoísmo; esto lleva a cada cual (sean agentes o árbitros) a pensar sólo en su propio sistema de utilidades, aun cuando su decisión afecte la confianza general, e incluso cuando de hecho lesione a otros miembros del colectivo. Dichos comportamientos individuales tienen desde luego la potencialidad de ser educables, y si se los estimula adecuadamente, las modificaciones que se logren en el nivel individual son por ellas mismas suficientes para producir cambios en la trama colectiva. El estímulo de las virtudes de honradez y altruismo no sólo lograría un cambio positivo en las propias conductas, sino que al mismo tiempo produciría una transformación virtuosa del escenario de las decisiones públicas.

También localizando el origen de las transgresiones en rasgos del comportamiento individual, otras posiciones centran más su atención en la segunda naturaleza humana llamada cultura, la cual de manera gradual va construyendo una tendencia hacia el ejercicio de las virtudes en unos casos o hacia la práctica de los vicios en otros. En el primer supuesto se generan resultados de bajos costos de transacción, mientras que en el segundo tales costos son elevados. Aquí la dicotomía vicio-virtud permanece, aunque se la localiza en el nivel cultural de los individuos; para corregir estos vicios culturales los procesos serían mucho más lentos que los de educación, ya que implican la remoción de capas que se han ido sedimentando pacientemente a lo largo del tiempo. Sin embargo, desde este punto de vista las transformaciones que se logren en el terreno cul- 
tural serían también suficientes para garantizar el fortalecimiento de los arreglos normativos que ese grupo humano produzca.

En las concepciones que localizan el origen de los problemas de fragilidad normativa en las conductas de los individuos, el destino de las decisiones públicas está asociado a esos comportamientos, por cuanto ellas no hacen sino reflejar cl carácter virtuoso o vicioso de los miembros del grupo. Desde este supuesto, ya sea que el mismo esté erigido sobre rasgos personales o culturales, la raíz de la fortaleza o debilidad de las tramas normativas se encuentra anclada siempre en el nivel de los individuos. Las eventuales modificaciones destinadas a producir efectos en el sístema de relaciones sociales deberían, desde esta perspectiva, incidir bien sea sobre los procesos de educación de los comportamientos o sobre la transformación de los patrones culturales de esas individualidades.

Sintetizando, tendriamos un primer método para disminuir el número de transgresiones que debilitan la trama de los arreglos normativos que se afinca en el concepto general de hacer que tales transgresiones resulten tan costosas como para desalentar nuevos casos. Este método considera que el sujcto acata las decisiones públicas desde un punto de vista externo, en virtud de un cálculo de utilidades. Los mecanismos de implementación de este método han puesto en evidencia su alto costo, y una clara ineficiencia. Ante csta ineficiencia y alto costo de las soluciones que se apoyan en un punto de vista externo, surgen grandes esperanzas cada vez que se habla de soluciones que se basan en el poder del comportamiento individual, y apelan a su punto de vista interno. Sin embargo, a pesar de que sería muy tranquilizador asignar a tales esperanzas una alta probabilidad de ocurrencia, los análisis muestran que actualmente no parece razonable suponer que desde el solo nivel individual sea posible resolver de una manera estable cl profundo dilema que enfrenta a las funciones de utilidad individual entre sî y a las funciones de utilidad individual con las funciones de utilidad colectiva, dilema que se escenifica en el diseño de las decisiones públicas.

Hay que señalar, sin embargo, que ambas posiciones han contribuido de manera sustancial a fortalecer el análisis de los costos de transacción asociados al diseño de arreglos normativos. Los métodos que se inclinan por decisiones públicas que sean capaces de controlar las transgresiones aumentando el costo de las mismas han logrado poner en evidencia importantes aspectos del cálculo de utilidades que realizan los transgresores, lo que ha permitido drásticos avances en el terreno de la psicología de la transgresión ${ }^{10}$. Asimismo, ha producido una herramienta eficaz para el análisis de los costos/beneficios derivados del acto de transgredir, $y$ de los mecanismos para su control. Paralelamente, los métodos que consideran que para el eficaz desempeño de los arreglos normativos hay que contar con un capital individual capaz de asumir un punto de vista interno en relación con las instituciones, ha servido para poner en evidencia el importante papel que ese punto de vista interno juega en la estabilidad de las soluciones normativas. Los métodos señalados han contribuido a la correcta formulación de un problema que pone en evidencia la necesidad de intentar la convocatoria del punto de vista interno en los usuarios de los arreglos normativos. También en este punto el utilitarismo contemporáneo ha producido fructíferas consecuencias.

Tal como se señaló inicialmente, los arreglos normativos nacen de actos de decisión normativa, que involucran tres componentes: (AN), que es el agente que produce la decisión; $(N)$, que es la pieza normativa que consagra el arreglo, y (S), que es el sujeto hacia el cual se dirige el arreglo. También se señaló que los tres más importantes problemas en el diseño 
de arreglos normativos son los de definición de las reglas, de establecimiento de los mecanismos de resolución de controversias, y de control de las transgresiones. Por regla general, quicnes se han dedicado al estudio del tema han abordado los problemas de costos/beneficios considerando que este punto sólo involucra la relación entre (N) y (S), es decir, que el diseño de las decisiones públicas debe producir arreglos tales que incidan eficazmente sobre los comportamientos de los sujetos; con este fin se han ideado numcrosos recursos instrumentales para perfeccionar el lenguaje, mejorar los sistemas de monitoreo, y diseñar sistemas punitorios que hagan que las transgresiones sean un mal negocio. El presupucsto implícito de tal enfoque es el siguiente: cuanto más se pcrfeccione técnicamente el diseño de los arreglos normativos, su incidencia sobre los comportamientos será más eficaz ${ }^{11}$. Esto implica aceptar que las «reglas clarass, la epronta acción de la justicia», la existencia de «métodos alternativos de resolución de conflictos» son equivalentes a un bajo costo de transacción y en consecuencia a una alta eficacia normativa. Sin embargo, cuando se incluyen en la consideración los costos derivados de las recursivas actividades de control y de sanción, como así también los costos implícitos atribuibles a la inestabilidad de las soluciones, la relación costo/beneficio sufre cambios demasiado sustancjales como para ignorarlos. En este punto hay que tener presente que incrementar la probabilidad de que una transgresión sea efectivamente castigada a fin de hacerla poco rentable impone a su vez un alto costo a la autoridad que decide, y a la sociedad en su totalidad $^{12}$. Y como los controles sobre controles se multiplican en forma exponencial, los costos que ellos producen pueden llegar a superar los beneficios que generan, lo cual debilita la eficacia de la solución, y desde luego atenta contra su estabilidad. Tal como se ha señalado, en las soluciones que ven a los arreglos normativos como meros mecanismos de corrección de conductas sólo se tienen en cuenta los componentes $(\mathrm{N})$ y (S) y, en consecuencia, centran su actividad en producir una relación eficiente entre ambos componentes. Este enfoque conductual del punto es por su propia definición incapaz de funcionar sin un costo importante asignado a una larga cadena de controles y sanciones.

A fin de superar este juego vicioso que lleva a pagar altos costos de represión para así aumentar el costo de las transgresiones, hay que pensar en el papel que debe jugar la autoridad normativa ( $A N$ ) en lo relativo a la construcción de un punto de vista interno en los usuarios de la decisión, punto de vista que los lleve no sôlo a corregir sus comportamientos en virtud de un cálculo de utilidades, sino en base a la convicción de la corrección normativa del arreglo. El logro del punto de vista interno es la máxima prueba de eficacia de un arreglo normativo. Esto es así porque por una parte tal logro tiende a hacer mínimos los costos de control y de sanción, y por la otra genera estabilidad en la solución normativa al ampliat la base de sustentación de dicha solución.

Para llevar a buen puerto la construcción del punto de vista interno es necesario considerar que la eficacia del arreglo normativo no depende de un solo aspecto, sino de dos. En efecto, generalmente se considera que la cficacia de los arreglos normativos tiene sólo que ver con la elaboración de un cuerpo de incentivos que provoque en los destinatarios de las normas una conducta de acatamiento basada estratégicamente en la relación (I); este error deriva de que sólo se considera que la institución descansa sobre el vínculo que se establece entre $(N)$ y $(S)$. La propia indole de la relación (I) pone en evidencia que este camino sólo apela al punto de vista externo, y que en consecuencia el arreglo normativo es acatado por (S) únicamente buscando su propio beneficio, pero no por- 
que él mismo lo considere deseable o correcto. Al estar la conducta de acatamiento sólo basada en un cálculo estratégico, el mismo es necesariamente inestable, ya que si (S) descubre mediante cualquier recurso el modo de bajar el costo que le produce una sanción, dejará automáticamente de acatar la regla. Para lograr tal estabilidad es necesario que los usuarios de los arreglos normativos asignen a los mismos un valor que no dependa exclusivamente del cuerpo de incentivos, aunque sî los comprenda. Para ello es necesario ampliar el concepto de eficacia; además de la clásica visión que define la eficacia en términos de la capacidad de los arreglos normativos (N) para producir modificaciones conductuales en los sujetos (S) es nccesario incluir la consideración de la relación entre la decisión de la autoridad de la cual emana la norma (AN) $y(N)$. En estos términos la eficacia de $(\mathrm{N})$ quedaria definida en base a dos componentes, ambos necesarios pero ninguno suficiente:

1. El que concierne a la relación entre los arreglos normativos (N) y los comportamientos de los sujetos a los que va dirigido el arreglo (S). En este aspecto, el diseño de un apropiado cuerpo de incentivos puede llegar a producir importantes modificaciones conductuales basadas en el acatamiento, las cuales abrirían el camino al desarrollo del punto de vista interno, aunque no serían suficientes para dotarlo de estabilidad.

2. El que concierne a la relación entre la autoridad normativa (AN) con (N). Esta relación se vincula al nivel de legitimidad y sustentabilidad de la decisión de (AN), el cual se asocia no con el acatamiento, sino con la receptividad de (N) por (S). En este aspecto lo que requiere de justificación es el valor normativo sobre el que se apoya la decisión de (AN), la cual reivindicará una vocación de corrección razonablemente aceptable por los sujetos. Esta inclusión de la evaluación del valor normativo en la estructura de justificación de las decisiones normativas, a pesar de requerir de una importante inversión inicial, ha demostrado ampliamente una enorme capacidad para abaratar los costos de transacción en la aplicación de dichas decisiones y un gran poder de estabilización del punto de vista interno.

\section{Contribuciones del utilitarismo} contemporáneo que refuerzan la eficacia normativa de las decisiones públicas

Según se señaló anteriormente, la eficacia normativa de una decisión está asociada con su capacidad para convocar en los usuarios la aceptación interna de su contenido normativo; a su vez, la presencia de esta propiedad se vincula directamente a la transparencia con que la decisión es elaborada.

Las contribuciones del utilitarismo contemporáneo en materia de favorecer la eficacia normativa a través del aumento de la transparencia de los procesos decisorios se manifiestan en tres aspectos: en primer lugar, queda establecido un modo específico de plantear el problema de decisión; en segundo lugar, se definen los mecanismos válidos de construcción de la decisión; y por último, se delimita la estructura de justificación de la decisión que puede ser legítimamente utilizada.

En relación con el modo de plantear un problema de decisión pública el utilitarismo señala que el mismo no puede ser considerado como un enfrentamiento entre posturas valorativas incondicionales, sino como un conflicto de vectores de utilidad en el cual el decisor va a necesitar una regla de decisión, o un mecanismo capaz de orientar la construcción de la decisión normativa. Desde este punto de vista, por ejemplo, un grupo ccologista que aboga por preservar intacta la naturaleza no será considerado incondicionalmente como el defensor por excelencia del equi- 
librio de la cadena biológica (aunque en efecto puedan serlo en una cierta medida), sino como unos actores sociales que tienen preferencia por el vector $\mathrm{U}_{\mathbf{l}}$, mientras que los agricultores que quieren talar bosques y hacer desaparecer especies salvajes para que nazcan praderas no serán descritos incondicionalmente como productores que buscan dotar de alimentos a la sociedad para disminuir el hambre (sin desconocer que en efecto cumplan esa función), sino como un grupo social que muestra preferencia por el vector $U_{2}$, bajo el supuesto de que ambos vectores son eficientes, es decir, ninguno de ellos es dominante. Estos vectores representan las preferencias especificas de cada grupo individualmente considerado; y el decisor debe construir un vector $B$ que busca integrar tales preferencias. Este último vector difiere de los anteriores en que mientras los vectores $U_{i}$ representan siempre el punto de vista de preferencias particulares de individuos o grupos, los vectores $B_{i}$ aspiran a expresar una solución general y estable al problema de integración de los vectores antagónicos $\mathrm{U}_{1}$ y $\mathrm{U}_{2 \text { - }}$

El hecho de situar el problema en un escenario que hace énfasis en la integración de los vectores antagónicos o en disidencia contribuye a evitar las dificultades que se derivan de las calificaciones deónticas incondicionales; sin embargo, aun dentro del marco favorable definido por esta nueva forma de plantear el problema de decisión, el decisor debe hacer frente a importantes escollos que emergen del propio carácter de las funciones de bienestar colectivo.

En efecto, cuando cl decisor público se enfrenta al problema de integrar en una única función de bienestar colectivo los vectores de preferencia individual sin violentar los principios de coherencia racional, debe resolver las tensiones que siempre exhiben las relaciones entre la racionalidad individual y la social. En este punto hay que considerar que si bien cuando nos enfrentamos a un problema que sólo involucra la racionalidad individual (teoría de la decisión individual o en situaciones de juegos), la regla de maximización de las expectativas de utilidades representa en sí misma un buen argumento para justificar la decisión; cuando tal decisión se plantea en el marco social, siempre surgen conflictos entre la racionalidad individual y la social. Estos conflictos son muy naturales, ya que la decisión pública establece necesariamente algunas restricciones a los vectores individuales de preferencias ${ }^{13}$.

En razón de estas inevitables tensiones entre el interés individual y el colectivo, es extremadamente importante dotar a las decisiones públicas de la mayor transparencia posible, no sólo para que las mismas desplieguen una sólida base justificatoria, sino para que los factores que fueron considerados en la decisión sean susceptibles de un análisis crítico, y de esta manera exista efectivamente la posibilidad de evaluar la responsabilidad del decisor. Este punto es particularmente importante, ya que en el marco de las decisiones públicas es inevitable dilucidar la espinosa cuestión de qué y cuánto de nuestro vector de utilidad estamos dispuestos (o podemos) conceder en favor del logro de la solución, la cual suclc no estar directamente asociada a nuestros propios intereses. Sea cual fuere la regla utilizada, toda decisión sobre bienes comunes es necesariamente restrictiva de los vectores de preferencia individual, y demanda de los actores alguna limitación a sus expectativas de utilidad. $\mathrm{Si}$ esto es así, y los individuos tienen que renunciar a porciones, a veces importantes, de su función individual de preferencia, no es extraño que tiendan a ser altamente exigentes a la hora de evaluar las razones que el decisor expone para justificar su selección. A causa de esta circunstancia, no es infrecuente que el decisor se encuentre en medio de un fuego cruzado entre actores, cada uno de los cuales está convencido de que las restricciones sufridas 
en su vector de preferencias son excesivas $o$ injustificadas.

Frente a tales situaciones de conflicto, que si no son bien resueltas pueden traer como consecuencia el debilitamiento de la capacidad normativa de la decisión con el consiguiente perjuicio para la trama colectiva, el decisor debe procurar fortalecer su base argumental mediante el despliegue metódico de las operaciones que lo guiaron en su decisión. Pero más allá de su función justificatoria, este despliegue tiene también la virtud de permitir al propio decisor incorporar a su solución el mayor número de elementos informacionales, con el fin de contribuir a la corrección de sus probabilidades subjetivas.

Desde este punto de vista, el problema más importante que debe resolver el decisor en materia pública deriva de que estas decisiones, además de integrar la existencia real de sistemas de preferencias en disidencia, tienen como finalidad intrínseca la de favorecer en los destinatarios de la solución el desarrollo de un punto de vista interno en relación con la decision. Por esta razón, este tipo de decisiones conciernen a la Ética y no a las Matemáticas, ya que la sola aplicación de una regla mecánica de maximización de las preferencias individuales no puede proveer de una justificación suficiente. La integración de los vectores en disidencia requiere de una perspectiva independiente ${ }^{14}$, que permita efectuar ponderaciones de los componen. tes vectoriales y al mismo tiempo contribuya a hacer más transparentes y discutibles las bases de tal operación. De este modo se perfecciona la estrategia de presentación del problema, y en consecuencia también se incrementan las probabilidades de lograr una solución más aceptable por Ios individuos.

El segundo frente a través del cual el utilitarismo contemporáneo logra favorecer la transparencia de las decisiones públicas es el de la definición de los mecanismos válidos de construcción de la decisión. La propia manera de plantear los problemas de decisión pública, que es característica del utilitarismo contemporáneo, tiene como consecuencia la necesidad de aceptar y realizar intercambios de utilidades en el escenario de la construcción de la decisión pública. Como las funciones individuales de utilidad que están en disidencia deben ser articuladas en una única pieza normativa, resulta inevitable que se lleven a cabo procesos en los cuales cada individuo o grupo concede parte de sus expectativas de utilidad a cambio de algo que considera equivalente. En este tipo de proceso no hace falta medir las utilidades sobre un mismo continuo, tema que ha preocupado a muchos pensadores ${ }^{15}$, ya que las unidades de utilidad no son, tal como se ha visto, unidades del tipo "peras" o «manzanas", y resulta perfectamente aceptable que un vegetariano asigne a tres manojos de berros la misma utilidad que un carnivoro le otorga a $200 \mathrm{gr}$. de lomito, y que en consecuencia ambos actores estén dispuestos a intercambiar tales bienes por considerar que los mismos satisfacen sus respectivas preferencias. Esta satisfacción de las preferencias, como puede verse, no está necesariamente asociada al valor monetario de los bienes en cuestión.

$\mathrm{El}$ problema fundamental que surge en estos procesos de intercambio es que en principio, y con una utilidad medida cardinalmente, las soluciones posibles son infinitas, lo que golpea duramente la operatividad del procedimiento. Para atacar este problema ha resultado fundamental la contribución de John Nash, quien en su artículo «The Bargaining Problem», publicado en 1953, logra establecer que bajo un conjunto de restricciones es posible encontrar una solución única a los problemas de negociación (como intercambio de utilidades individuales). Apoyándose en la construcción conceptual denominada «frontera Pareto-óptimo» que permitió acotar el espacio de las soluciones posibles en procesos de negociación, Nash logra 
establecer que en esa frontera se puede obtener una única solución en un problema de intercambio de utilidades. Tal «solución" consiste por una parte en un conjunto de restricciones que delimitan los procedimientos algebraicos mediante los cuales se llega a la solución; por otra parte, la solución Nash señala también lo que podría considerarse como la meta u horizonte de los procesos de negociación ${ }^{16}$. En este último sentido la solución marca la existencia de un punto hacia el cual los procesos de intercambio deberían tender.

Aunque el citado esquema es válido para los procesos típicos de intercambio de utilidades individuales, las cosas se complican drásticamente en el caso de la construcción por parte de una autoridad normativa de una función de utilidad colectiva, ya que aunque en efecto se trata básicamente de intercambios de utilidades, las soluciones aspiran a ser asimismo normativamente correctas ${ }^{17}$. Un decisor público debería, en consecuencia, preocuparse por las posibles asimetrias iniciales en el escenario de negociación, las cuales podrían dar lugar a formas de cxplotación de un actor por otro. Bajo estas circunstancias el decisor público opera necesariamente como un árbitro, y en tal condición la aplicación lisa y llana de la regla utilitarista de maximización de las utilidades esperadas no le permite discriminar entre la calidad de las pretensiones individuales en juego. Ésta, sin dudas, no es una cuestión ética de menor cuantía; si el arreglo aspira a ser normativamente eficaz, el decisor que efectúa el arreglo está en la obligación de fundamcntar la pretensión normativa de dicho arreglo, y de las reglas que del mismo se derivan.

Antes de proceder a la maximización de las utilidades que expresan las preferencias individuales, quien construye una decisión pública tiene que ponderar tales preferencias sobre la base de un marco que naturalmente constituye una forma de regla externa a tal sistema. Esta regla, que de algún modo impone limitaciones a la competencia del decisor, no puede ser de base exclusivamente empírica, aunque de ningún modo puede ignorar la empiria; tampoco puede ser sólo de base lógico-deductiva, debiendo sin embargo respetar los principios de consistencia, y debe ser susceptible de crítica racional y evaluación en ambos aspectos. Sobre esta base informacional no todos los intercambios son igualmente deseables, aunque el intercambio de utilidades continuará siendo la herramienta de instrumentalización por excelencia.

En el caso anteriormente mencionado de los ecologistas y los agricultores, aun cuando sea perfectamente coherente intercambiar en una decisión pública una unidad de bosque por una unidad de pradera (cosa que se hace toda vez que se determina la dosis óptima de un fármaco), a la hora de legitimar y justificar ese intercambio el decisor no podrá ignorar que los estudios científicos realizados en nuestro siglo han demostrado la fragilidad del equilibrio ecológico, el cual define límites a su competencia de decisión, más allá del problema inmediato a resolver. Tampoco podrá ignorar que aunque las grandes hambrunas han sido y son el método de control ccológico del crecimiento poblacional para todas las especies, el hombre ha establecido nuevas reglas, que por una parte han alterado desde el comienzo el primitivo equilibrio natural, pero gracias a las cuales hay en términos generales cada vez menos sufrimiento, mayor esperanza y mejor calidad de vida.

Este marco, en efecto, restringe la competencia del decisor, pero no de la manera en que lo haria la prohibición incondicional de un cierto tipo de acciones; se trata más bien de una suerte de insumo informacional cuya consideración no podría obviarse sin el riesgo de que la pretensión normativa de decisión se hiciera altamente vulnerable. En cuanto al contenido de este marco, no habría posibilidad alguna de eli- 
minar del mismo elementos universalizables de base deontológica, como tampoco habría modo de evitar la inclusión de información de base empírica o lógica.

Tal como se ha podido notar a lo largo de la exposición, cuando se habla de decisiones en el entorno colectivo aparecen constantemente expresiones tales como probabilidad de ocurrencia, niveles probables de degradación ambiental o expectativa de vida. Esta recurrencia no debe parecer extraña, ya que si se elimina el enfoque incondicional que define tipos de actos prohibidos o permitidos dibujados con plena certeza, entra inmediatamente en escena la incertidumbre, $y$ su modelo axiomätico asociado: la probabilidad.

Sin embargo, si sólo se pudieran construir las utilidades y tomar las decisiones cuando se cuenta con probabilidades objetivas $^{18}$, las decisiones quedarían condenadas a la rigidez, e importantes esferas (como la valorativa) excluidas del escenario decisional. Esto llevó a los utilitaristas contemporáneos a retomar el uso de las llamadas probabilidades subjetivas, las cuales se basan en la apreciación subjetiva que el decisor, sobre una base informacional amplia y versátil, asigna a un determinado resultado. Estas probabilidades subjetivas, aunque se basan inicialmente en el llamado «grado de creencia», son susceptibles de tratamiento numérico, y satisfacen un sistema de axiomas muy próximo al de las probabilidades objetivas ${ }^{19}$.

Desde el punto de vista de la plausibilidad de sus axiomas, habría que señalar que las probabilidades subjetivas reflejan de manera acertada cl comportamiento más habitual de los decisores públicos, quiencs sobre la base de su experiencia, sistema de valores $\mathrm{c}$ información preliminar, formulan una distribución probabilística a priori, la cual representa su grado de creencia acerca del comportamiento del parámetro, antes de recabar datos adicionales ${ }^{20}$. En to que se ha definido como experiencia, sistema de valores, $e$ informa- ción previa, se articulan de una manera compleja la información de base empirica o experimental, la de base conceptual o teórica, junto a calificaciones a priori de base deontológica incorporadas al sistema general de valores del que el decisor participa.

Especialmente debido a los lúcidos y sistemáticos desarrollos matemáticos de Thomas Bayes ${ }^{21}$, se cuenta hoy con métodos axiomáticos para la actualización de una distribución a priori, la cual gracias a tales métodos puede ser convertida en una distribución a posteriori a la luz de toda nueva información relevante al problema de decisión que se está considerando. En este contexto, la corrección sucesiva de las distribuciones probabilísticas se constituye en la herramienta clave para la elaboración y justificación de las decisiones públicas.

El uso sistemático de esta herramienta impone una mayor exigencia de transparencia a las decisiones públicas, por cuanto cada pieza de información considerada relevante para el asunto bajo consideración tendrá que ser incorporada en la función de probabilidad, y en consecuencia producirá una modificación en la estimación del parámetro. $\mathrm{Si}$, por el contrario, el decisor no considera neccsario incorporar tal información, estará en la obligación de justificar dicha omisión con razones suficientes. Como se puede apreciar, este enfoque de las probabilidades subjetivas a priori y el método bayesiano de corrección de las mismas, despliegan de mancra mucho más diáfana el proceso de construcción de las decisiones, y en consecuencia permiten ejercer de una manera más activa la función de control sobre las mismas.

La transparencia que produce en la construcción de las decisiones la corrección bayesiana de las probabilidades subjetivas a priori, permite ver detalladamente la traza de todo el proceso; esto tiene una consecuencia de vital importancia al momento de evaluar la calidad de la jus- 
tificación con la que se pretenda sostener dicha decisión. En efecto, bajo este procedimiento no tiene cabida el estilo uprimero decido y luego busco la justificación más adecuada a mi decisión», que suele ser de empleo frecuente en este terreno; aquí, tanto las piezas informativas que quedan incorporadas como las que fueron excluidas constituyen la base argumental única sobre la cual puede fundarse una justificación para ser aceptable ${ }^{22}$.

El estilo bayesiano de construcción de las decisiones permite leer en las probabilidades subjetivas a prioni las bases deónticas, las intuiciones, los conocimientos y los sentimientos morales en los que se origina la decisión; mientras que el proceso de recursiva conversión de tales probabilidades $a$ priori en probabilidades a posterioni nos muestra las sucesivas correcciones que la decisión va sufriendo, y exhibe las razones que fundamentan cada paso del proceso.

\section{Conclusiones}

El acucioso lector ya ha advertido en los cinco apartados que integran el artículo que, bajo una apariencia ordenadamente descriptiva, he articulado un discurso en el que asigno a los hallazgos del utilitarismo contemporáneo un valor positivo para la ética de las decisiones públicas. Para ser consistente con mi creencia de que las asignaciones de valor son sólo interpretables en un marco específico que cual sistema de coordenadas permita establecer el espacio de validez de la asignación, tengo que hacer explícitos los hasta ahora implícitos supuestos desde los cuales he elaborado mi juicio valorativo.

1. Las decisiones públicas son técnicamente decisiones bajo condiciones de incertidumbre. La incerlidumbre de estas decisiones deriva tanto de la esfera valorativa como de la fáctica y la del comportamiento de los actores sociales. Esto implica que las probabilidades que pueden asignarse en el marco de estas decisiones corre entre 0 y 1 , excluyendo los extremos.

2. Las decisiones públicas afectan las llamadas cosas comunes, ya se trate de bienes, espacios o sistemas de valores. En el ámbito de estas cosas comunes, las decisiones públicas atribuyen status deóntico a las acciones (prohibidas, obligadas o permitidas), o efectúan asignaciones distributivas de cargas o beneficios. Para cumplir su objetivo, las decisiones públicas establecen arreglos normativos que imponen restricciones al dominio privado.

3. La calidad de una decisión pública se juzga por la consistencia de su estructura formal, por la capacidad de su sistema de incentivos para lograr el control de los conflictos entre funciones de utilidades en disidencia, y por su eficacia en la promoción del llamado punto de vista interno entre los destinatarios de la normatividad.

Analicemos entonces los hallazgos del utilitarismo en el marco de los supuestos señalados. Si se aceptan las condiciones establecidas en 3 como los criterios de evaluación de una decisión pública, hay que reconocer que el utilitarismo contemporáneo ha contribuido a fortalecer todos los aspectos de las decisiones públicas de manera sustancial. En materia de consistencia formal, el contar con un cuerpo de axiomas y la definición completa de la estructura primitiva ha permitido no sólo producir decisiones evaluables al interior de su propio sistema lógico, sine también con una mayor capacidad de ser comparadas con soluciones alternativas; pudiendo asimismo ser internamente criticadas en base a un criterio sólido de corrección. En relación con el segundo criterio de evaluación de las decisiones públicas, es decir, el relativo a su capacidad para construir un sistema de incentivos con eficiencia en el manejo de las disidencias, las posibilidades que ofrece el utilitarismo contemporáneo son francamente importantes; ellas se derivan de la potencialidad que 
brinda el concepto de utilidad de Von Neumann-Morgenstern para el manejo sutil de los sistemas de punición-recompensas, y la capacidad que ese mismo concepto unido al de probabilidad subjetiva posee para la estimación de escenarios que facilitan el cálculo de consecuencias. Finalmente, si se toma en consideración que las decisiones públicas, además de ser evaluadas en su capacidad para controlar las disidencias mediante los sistemas de incentivos, deben probar también su eficacia normativa, el aporte del utilitarismo cobra una mayor dimensión. En efecto, pareciera que no cxiste un mejor recurso para convocar el punto de vista interno en los destinatarios de los arreglos normativos que el que ofrece la transparcncia y el uso sistemático de las probabilidades subjetivas como mecanismos de elaboración de las decisiones.

Cuando los supuestos 1 y 2 se exhiben uno al lado del otro con toda su contundencia producen generalmente un efecto de gran desasosiego entre quienes se dedican al estudio y a la construcción de decisiones públicas. Aceptar como punto de partida que las decisiones públicas se toman bajo incertidumbre, y que ellas sirven para imponer restricciones al dominio individual, no resulta en verdad nada tranquilizante. A pesar de que intuitivamente se percibe la plausibilidad de estos supuestos, no deja de producirse la impresión inicial de que en relación con algo que puede afectarnos profundamente no sólo en nuestro actual sistema de utilidades, sino en lo que ello significa para nuestros planes de vida, somos colocados inexorablemente en manos de aprendices de brujos, cuyas inciertas metodologias no les impiden tener un amplísimo poder sobre los aspectos más relevantes de la vida social. Este sentimiento, $y$ la profunda inseguridad que el mismo conlleva, es uno de los factores que más inciden en la aceptación de otros supuestos, que, aunque menos plausibles, son capaces de transmitir un mayor nivel de confianza. Sin duda, es más alentador suponer que quienes construyen las decisiones públicas poseen certeza acerca de cuáles son los fines más valiosos, que estos fines son en efecto determinables, y que hay una abundante disponibilidad de precisas herramientas para alcanzarlos. Sin embargo, cuando un decisor asume un supuesto que es negado sistemáticamente por los hechos, la calidad de sus decisiones se ve debilitada. Aunque suponer que una torre de control de vuelos está dotada de un servicio de radar moderno y operativo puede resultar tranquilizante para la tripulación y el pasaje de una aeronave, si tal supuesto está negado por la realidad, el asumirlo incrementa notablemente la probabilidad de sufrir accidentes en el aterrizaje, ya que impide desarrollar métodos alternativos de orientación del descenso.

Cuando se acepta el supuesto de incertidumbre (incertidumbre puede derivarse de la información imperfecta o incompleta), todo esfuerzo que permita mejorar la información incorporada a la decisión incrementa simultáneamente la calidad de la decisión. En este punto el refinamiento del concepto de utilidad realizado por Von Neumann-Morgenstern dota a la información acerca de las preferencias de un alto nivel de precisión, lo cual permite mejorar notablemente las bases instrumentales de las decisiones. Adicionalmente, como las decisiones basan sus asignaciones deónticas y distributivas sobre el intercambio de las utilidades de los agentes afectados por la decisión, el contar con tales bases instrumentales permite una mayor versatilidad y precisión en tales intercambios, to que contribuye a que los mismos puedan ser mejor interpretados y aceptados por aquellos cuyas utilidades se ven restringidas.

A pesar de la importancia de todas las contribuciones señaladas y de su innegable incidencia sobre la calidad de las decisiones públicas, su fuerte carácter instrumental puede alimentar dudas acerca de si las mis- 
mas conciernen efectivamente a la «ética» de las decisiones públicas. Para dilucidar este punto es conveniente recordar que en sus aspectos normativos las decisiones elaboradas bajo condiciones de incertidumbre carecen del a la vez monolítico y frágil soporte que puede ofrecerle el punto de vista deontológico, el cual asume incondicionalmente un determinado valor (con probabilidad 1 ó 0 ); tampoco pueden basarse completamente en las llamadas soluciones algebraicas; en consecuencia, tales decisiones tendrán que construir su propia estructura de legitimación mediante procedimientos que no "postulen» corrección normativa, sino que la amuestren». Esta kexhibición» hecha ante sujetos a los cuales se afecta en sus utilidades entraña grandes dificultades. En primer lugar, para el utilitarismo tales sujetos son plenamente autónomos, ya que los ha reconocido como capaces de ordenar coherentemente sus preferencias, lo cual implica que se hacen cargo de todas las consecuencias de sus ordenamientos; es decir, que son sujetos morales. Desde este punto de partida, el utilitarismo no podría aceptar sin una sólida justificación que una decisión pública ignore ciertas preferencias 0 las perjudique en el intercambio de utilidades ${ }^{23}$. Sin embargo, el decisor público tampoco podría ignorar piezas de información relevantes a su decisión ${ }^{24}$, ya que éste es precisamente el aspecto central del método bayesiano de corrección de probabilidades. Desde un punto de vista lógi$c o$, estas dos condiciones están en conflicto, ya que la primera indica asignar a todas las funciones de utilidad individual el mismo peso en la función de utilidad colectiva, mientras que la segunda indica introducir correcciones a las probabilidades en base a toda información relevante al problema de decisión. La exístencia de esta contradicción impide producir las decisiones públicas mediante algoritmos, por lo que el decisor público debe operar como un árbitro, lo cual supone privilegiar un curso de acción sobre otro; pero esto es bastante natural, ya que el aplicar el arbitrio para escoger entre una solución u otra constituye el núcleo mismo de todo proceso de decisión. Como puede verse, ningún mecanismo algebraico ni asignación deóntica incondicional puede modificar el hecho de que los procesos de decisiones públicas sean procesos de arbitraje que por definición ticnen un componente valorativo.

Este es el complejo marco (despojado de la relativa tranquilidad que ofrecen los prejuicios incondicionales) en el que las decisiones públicas deben «mostrar» su vocación de corrección normativa; y es en este punto en el que los avances realizados por el utilitarismo, siendo fuertemente instrumentales, atañen sin embargo de manera directa a la ética de las decisiones públicas. En el momento de llevar adelante la construcción de la decisión, la condición que impone la obligatoriedad de corregir las probabilidades subjetivas a priori con las piezas informacionales relevantes a la decisión, siendo básicamente instrumental, permite incorporar al proceso decisional elementos de base tanto fáctica como valorativa, que enriquecen el paisaje moral de la decisión. Por su parte, la condición que establece el uso de métodos sistemáticos de corrección de las probabilidades obliga a «exhibir» el origen de las ponderaciones valorativas que el decisor atribuye, atando la decisión a la responsabilidad, asunto éste básicamente ético. Por último, la condición que impide que las piezas informacionales que no hayan sido efectivamente utilizadas en la corrección de las probabilidades a priori puedan ser incorporadas en los argumentos de justificación de la decisión incide directamente en la calidad ética de esta justificación.

En sintesis, abandonada la nada plausible creencia de que el problema de construcción de decisiones públicas es limpiamente manejable desde un radar (deontológico o algorítmico), y aceptada la 
incertidumbre como el ambiente natural en el que tales decisiones se construyen. tenemos también que aceptar que todo mecanismo que contribuya a dotar a la navegación de formas racionales de corrección y control no sólo constituye un avance instrumental, sino que incide sustancialmente en las bases éticas de la decisión. Éste es el caso del utilitarismo contemporáneo.
I Puede verse J. Rentham, Official Aptitude maximized, Expense minimized, Oxford University Press, 1993, y A Protest against Law Taxes, P. Byme Ed, 1793.

z Cuando John Nash determina la existencia de una solución única al dlamado bargaining problem pudo pensarse que de alguna manera se habia encontrado un método para rcsolver todos los problemas de intercambio de utilidades; sin embargo, esta solución era válida sólo en el limite de las restricciones establecidas por Nash. Kalai y Smorodinski, al modificar una de las restricciones por Nash, Jogran otra «solución únicaw, Estc y numerosos ejemplos evidencian que el modelo formal que se utiliza incide de manera directa en la solución que se atribuye a un problema de decisión.

* Las decisiones públicas siempre implican la caracterización de tres elementos: los cursos de acción alternativos que de algún modo definen el espacio de decisiones posibles, las condiciones de los entornos ponderadas por su probabilidad de ocurrencia, y las utilidades esperadas. Como puede verse, todos estos clementos definen un escenario típicamente condicional.

+ La estructura primitiva incorpora los presupuestos Iógico-matemáticos de la tcoría de probabilidades, las definiciones primitivas especificas, y el sistema de axiomas.

5 La publicación de esta obra estuvo precedida por dos artículos seminales de Von Neumann; "Zur Theorie der Gesellschaftsspicle", publicado en Mathematische Annalen, 100, 1928, y uber cin oekonomisches Gleichungsystem und eine Verallgemeinerung des Brouwerschen Fixpunktsatzes", publicado en Engehnisse cines Mathematik Kolloguiums, 8, 1937.

- Una de las primeras consecuencias en este ámbito se expresa en el intluyente artículo de John Harsanyi, "Cardinal utility in welfare economics and in the theory of risk taking», publicado en 1953.

* Von Neumann y Morgenstern al considerar este caso establecen que las alternativas $B$ y $C$ sean mutuamente excluyentes, es decir, que la probabilidad de ocurrencia de una es complementaria de la probabilidad de ocurrencia de la otra.

3 Dos diferentes tratamientos axiomáticos de la utilidad se encuentran en los trabajos de I. N. Herstein y. W. Minor, "An Axiomatic Aproach to mensurable utilityn, Econometrica, 21, 1953, y R. Luce y H. Raiffa,
Gumes and Decisions, Wiley, NY, 1957. Analizando comparativamente ambos sistemas se puede apreciar claramente el motodo de trabajo señalado.

- Acerca de este punto puede verse R. Coase, «The problem of social Costs, Joumal of Law and Economics, 3, 1960, y E. Garzón Valdes, «Mercado y Justicia», Isonomia, 2, 1995.

10 Sobre cómo opera el análisis de costobencficio en la propensión a la transgresión puede verse J. Barragán, "Politieas y violencia», en el volumen Las libertades y sus encmigos, Ed. Cedice, Caracas, 1997.

"Sobre algunos puntos de vista en relación con los métodos de control de las transigresiones resultan especialmente ilustrativos E. Ostrom, Goveming the Commons, Cambridge University Press, 1990; V. Ostrom, D. Feeny y H. Picht (eds-), Rethinking Insitutional Analysis and Developntent, ICS Press, Ca., y R. Gooding (ed.), The Theon of Institutional Design, Cambridge University Press, 1996.

12 J. Barragán, «Decisiones nomativas y costos de transaccion*, en Revista del Banco Central, wol. XI, núm. 1, Caracas, 1997.

13 Algunos autores como Harsanyi consideran inicialmente que la construcción de la función de bienestar colectivo es el resultado de una opcración mate mática como la suma o la multiplicación, lo cual tiende a dar la falsa impresión de que las preferencias de los distintos actores son homogéneas; sin embargo, ante la clara evidencia de que no todas las preferencias son igualmente significativas pará la construcción de la función de bienestar colectivo (B) se ven obligados a ponderar tales preferencias (previo a sumarlas o multiplicarlas), lo cual implica admitir la existcncia de conflictos entre las mismas. Es obvio señalar que tales ponderaciones inciden de manera directa en el contenido de $B$.

${ }^{4}$ Se muy bien que esto lucirá altamente herético a los ojos de cualquier utilitarista ortodoxo, ya que el mismo podría suponer que por all se están infil trando quién sabe que indeseables demonios kantianos. Sin embargo, como no es concobible cn el utilitarismo contemporäneo la deliberada e incondicional renuncia a una fuente de información eventualmente relevante para la decisión, diffeilmente se podria justificar la negativa a incorporar una perspectiva que permite eva- 
luar y ponderar la calidad de los componentes vectoriales.

is Pueden verse, entre otros, las interesantes observaciones realizadas por $J$. Griffin en su obra On the Well Being, cuando se ocupa de las comparaciones interpersonales.

16 Los requisitos establecitos por Nash son: 1. Invariancia con respecto a la medida de utilidad; 2 . Optimo de Pareto; 3 . Simetria, y 4 . Independencia de las alternativas no relevantes. Acerca de las condiciones 1,2 y 3 , existe un acuerdo de aceptación muy generalizado, pero en relación con el requisito 4 se han escuchado voces disidentes. Sobre este punto puede verse en Sistemi Espenti nel Dinito mi artículo "Negoziazione e Incertezza", donde se analiza el alcance del concepto de solución, sobre la base de considerar las objeciones quc Kalai-Smorodinski han realizado al requisito 4 establecido por Nash.

"Sobre las limitaciones del concepto ksolución" desde el punto de vista éticomormativo puede verse el ya citado trabajo "Negoziazione e Incertezza».

${ }^{18}$ Se da el nombre de probabilidad objetiva a aquella en la que la estimación del comportamiento de un parámetro se efectía sobre la base de un número $\mathrm{N}$ de ensayos experimentales previos. Por su propia indo le, la probabilidad objctiva se adecua muy especialmente a las Ciencias Físicas, en las que los fenómenos obedecen a un comportamiento mảs o menos constante, lo que permite predecir con buen nivel de acierto el futuro a partir del pasado. En el caso del comportamiento humano, entre otros, la imposibilidad de trabajar con métodos experimentales trae como consecuencia la imposibilidad de utilizar exitosamente tales probabilidades.

19 Los axiomas de Kolmogorov introducidos en 1933 fijan las condiciones que deben ser satisfechas por las funciones de probabilidad objetiva. Expresados brevemente, cstos axiomas dicen así: $1.0<P(A) ; 2$. $P$ (de todos los posibles eventos) $=1$, y 3. Aditividad contable. De Finetti encuentra que sustituyendo el axioma 3 por el axioma 3 ' de Aditividad finita, es posible dar a las probabilidades subjetivas un tratamiento axiomático semejante al de las probabilidades objetjvas. Existen otros tratamientos axiomáticos que han merecido consideración muy especial como el de L. Savage, quien elabora un sistema apoyado en 7 axiomas, y el de A. Rényi, basado en 3 axiomas, el cual asume el carácter invariablemente condicional de la probabilidad.

2 El pleno uso de las probabildades subjetivas on la toma de decisiones se debe cn una gran medida a los trabajos de J. Harsanyi. En especial pueden con- sultarse: "Games with incomplete information played by "Bayesian" players" (1967/68), "Bayesian decision thcory, rule utilitarianism, and Arrow's impossibility thcorem (1979), $y$ «Decision and game theoretic models in utilitarian ethics" (1988).

21 Mientras que las ideas básicas que nutren este cnfoque son desarrolladas por James Bernoulli, su estructura matemática, factor fundamental para la realizabilidad de un sistema de calificaciones deónticas, se debe al Reverendo Thomas Bayes. El llamado Teorema de Bayes, como formulación matemática, nos dota del procedimiento formal necesario para modificar, en base a toda nueva información obtenida, nuestra creencia previa acerca del valor de un parámetro. Una información más extensa sobre este punto se pucde obtener en la obra de E. Pearson, The History of Statistics in the 17 th and 18 th Centuries.

32 Con el fin de establecer un nexo firme cntre ol proceso de construcción de la decisión y la justificación de la misma, he considerado oportuno establecer una condición (conocida como *Condición Barragán* ) que impide incorporar en los argumentos de justificación de la decisión cualquer pieza informacional que no haya sido oportunamente utilizada (por inclusión o exclusión) en la corrección de las probabilidades subjetivas a priori. De esta manera se busca garantizar que la justificación refleje ficlmente el proceso de ela. boración de la decisión, facilitando asimismo la adjudicación de responsabilidades.

${ }^{23}$ Encuentro, por ejemplo, que la eliminación que Harsanyi hace de las preferencias heteroorientadas y de las desinformadas, entre otras, cuando construye la función de bienestar colectivo, no se hace cargo de todo el impacto que tal hecho produce sobre la autonomía de los sujetos. Tampoco Binmore, quien critica la posición de Harsanyi, logra resolver csta contradicción.

24 En relación con la incorporación de información relcuante a la decisión, Barcón propone en su uTeoría de la Utilidad» un ejempla muy ilustrativo. Es el caso de un cliente que entra en un restaurante de regular aspecto y el camarero le informa que el menú contempla parrilla de solomo y filete de pargo. El cliente, aunque prefiere el pescado, selecciona la parrilla, basado en el a priori que le ha producido el aspecto del restaurante. Minutos después el camarero regresa para decirle que el chef le ha informado que también hay ancas de rana. "Muy bien - dice el clicnte-; sirvame el filete de pargo.s El cliente, con la nucva información cambió su a priori sobre la capacidad del restaurante para preparar el pescado. 